\title{
Factores de comorbilidad y secuelas de covid 19 en trabajadores de un hospital nacional de Huancayo 2021
}

Comorbidity factors and sequels of covid 19 in workers of a national hospital of Huancayo 2021

\begin{abstract}
Anahi Indira De La Calle Castro ${ }^{1, a}$, Rebeca Tello Carhuanca ${ }^{1, b}$, Teresa Leonor Villegas Félix ${ }^{1, c}$,
\end{abstract} Edith Suasnabar Cueva ${ }^{1, d}$

\section{RESUMEN}

Objetivo: Determinar la relación de los Factores de Comorbilidad y Secuelas de Covid 19 en trabajadores de un Hospital Nacional de Huancayo 2021. Material y Métodos: El método de esta investigación fue científico, de tipo básico, nivel descriptivo correlacional, diseño no experimental, de corte transversal. La muestra de estudio calculada probabilísticamente fue de 137 trabajadores. Resultados: El 52,6\% de los pacientes tuvieron COVID-19, la mayoría leve $(51,8 \%)$, y severo el $14,6 \%$, el $81,8 \%$ tuvieron contacto con los pacientes, el 58,4\% cree que se contagió en el hospital, recibió atención en su casa el $43,8 \%$ y en un hospital general el $29,9 \%$. La mayoría tenía entre 31 y 40 años $(35,8 \%)$ seguido de 41 y 50 años $(30,7)$, fueron de sexo masculino $(60,6 \%)$. Entre los factores de comorbilidad tenían: sobrepeso el $65 \%$, diabetes el $8 \%$, VIH $0 \%$, cardiopatías el $3 \%$ asma al 10,2\%, y enfermedades renales el $1,5 \%$. La mayoría no tenía secuelas del aparato respiratorio (40,9\%), tampoco cardiovasculares $(86,9 \%)$, ni secuelas psicológicas $(89,1 \%)$ tampoco dermatológicas $(47,4 \%)$. En relación a las secuelas neuropsiquiátricas se observa que tuvieron pérdida de olfato el 4,4\%, cefalea el 50,4\%; cambios de humor 15,3\%; disfunción neuromuscular 0,7\%; dificultad para pensar o concentrarse el $8.8 \%$; depresión 40,9\%; ansiedad 9,5\%; dolor muscular 10,2\%. Conclusiones: Existe relación entre los factores de Comorbilidad y secuelas relacionados con el Covid 19 en trabajadores de un Hospital Nacional de Huancayo 2021 ( $\mathrm{p}$ valor $=0,007<0,05$ ).

PALABRAS CLAVE: factores de comorbilidad, secuela COVID 19.

\section{SUMMARY}

Objective: To determine the relationship of Covid 19 Comorbidity Factors and Sequelae in workers of a National Hospital of Huancayo 2021. Material and Methods: The method of this research was scientific, basic type, descriptive correlational level, non-experimental design, cross-sectional. The study sample calculated probabilistically was 137 workers. Results: the majority professionals in $52.6 \%$, most of them had COVID-19, mild (51.8\%) and severe $14.6 \%, 81.8 \%$ had contact with patients and believe that it was infected in the hospital in $58.4 \%$ and $43.8 \%$ received care at home and in a hospital overall $29.9 \%$. The majority were between 31 and 40 years old (35.8\%) followed by 41 and 50 years (30.7), they were male $(60.6 \%)$. Among the comorbidity factors they had: $65 \%$ overweight, $8 \%$ diabetes, $0 \% \mathrm{HIV}, 3 \%$ heart disease, $10.2 \%$ asthma, and $1.5 \%$ kidney disease. Most did not have respiratory system

\footnotetext{
Universidad Privada de Huancayo Franklin Roosevelt. Huancayo, Perú.

Docente; Licenciada en Enfermería; Magister. ORCID ID: 0000-0003-1206-1321

Docente; Licenciada en Enfermería; Magister. ORCID ID: 0000-0003-3915-4405

Docente; Licenciada en Enfermería; Magister. ORCID ID: 0000-0001-8385-1596

Docente; Licenciada en Enfermería. ORCID ID: 0000-0002-2202-8720
} 
sequelae (40.9\%), nor cardiovascular (86.9\%), nor psychological (89.1\%) nor dermatological (47.4\%) sequelae. In relation to neuropsychiatric sequelae, it is observed that $4.4 \%$ had loss of smell, headache $50.4 \%$; mood swings $15.3 \%$; neuromuscular dysfunction $0.7 \%$; Difficulty thinking or concentrating $8.8 \%$; depression $40.9 \%$; anxiety 9.5\%; muscle pain $10.2 \%$. Conclusion: There is a relationship between Comorbidity and sequelae factors related to Covid 19 in workers of a National Hospital of Huancayo 2021 ( $\mathrm{p}$ value $=0.007<0.05$ ).

KEYWORDS: comorbidity factors, sequel COVID 19.

\section{INTRODUCCIÓN}

En el mundo, el siglo XXI se ha caracterizado desde sus inicios por una problemática de salud que ha afectado al mundo y Cuba no ha podido escapar de esta situación, que va desde un incremento de la resistencia microbiana, aumento de las enfermedades oncológicas hasta la aparición de nuevas enfermedades infecciosas emergentes y reemergentes, como ha sido la aparición de la COVID-19 a finales del pasado año. $(1,2)$ Los coronavirus son una extensa familia de virus que pueden causar enfermedades tanto en animales como en humanos. En los humanos, se sabe que varios coronavirus causan infecciones respiratorias que pueden ir desde el resfriado común hasta enfermedades más graves como el síndrome respiratorio de Oriente Medio (MERS) Tanto el nuevo virus como la enfermedad eran desconocidos antes de que estallara el brote en Wuhan (1).

La COVID-19 es una enfermedad infecciosa emergente, descrita por primera vez en diciembre del 2019 como un brote neumónico de etiología desconocida en Wuhan, China. Posteriormente, se identificó al nuevo coronavirus SARS-CoV-2 como agente causal y se demostró su transmisión entre humanos. En la mayoría de los casos la presentación clínica es leve e incluye síntomas inespecíficos como cansancio y fiebre; acompañados de tos seca, congestión nasal, rinorrea y dolor de garganta -similar a otros infecciones respiratorias-. Sin embargo, se han descrito signos y síntomas más diversos como diarrea, ageusia y anosmia; y en casos graves: neumonía, síndrome de dificultad respiratoria aguda, sepsis y choque séptico. El 30 de enero de 2020 la OMS declaró la enfermedad por coronavirus como una emergencia de salud pública de importancia internacional. A pesar de los subsecuentes esfuerzos de contención, el virus continuó extendiéndose en el resto de Asia y Europa y llega a América Latina el 26 de febrero de 2020, con el primer caso informado en territorio brasileño- Debido a su distribución, gravedad y el creciente número de casos fuera de China, la OMS declaró a la COVID-19 como pandemia el 11 de marzo. Al 16 de agosto de 2020 se han notificado más de 21000000 infectados y 760000 defunciones en todo el mundo, de los cuales aproximadamente el $54 \%$ de casos y defunciones se concentran en la región de Las Américas (2).

Según datos de la Organización Mundial de la Salud, cuatro de cada 10 personas que han fallecido por este padecimiento tenían sobrepeso, obesidad, hipertensión o alguna combinación de estas enfermedades. Un metaanálisis de las comorbilidades en estudios de China, sugiere que la hipertensión está presente en aproximadamente el $17 \%$ de los pacientes, la diabetes en el $8 \%$, las enfermedades cardiovasculares en el $5 \%$ y las enfermedades respiratorias en el $2 \%$ de los casos graves por coronavirus. Más aún, los pacientes ingresados en la unidad de cuidados intensivos (UCI) tuvieron un mayor número de comorbilidades $(72,2 \%)$ frente a los no admitidos en el UCI (37,3\%) (3).

En el Perú, el Centro Nacional de Epidemiología, Prevención y Control de Enfermedades reporta para el 10 de junio del 2020 una cifra de 6,109 defunciones con una letalidad del 2,83\%. Si bien se observa que la tasa de letalidad promedio del Perú es menor que el promedio mundial (5,7\%), en Lambayeque alcanzamos el $6,24 \%$ que es superada solo por el departamento de Ica en un $6.49 \%$. Estas cifras exponen la crítica situación de las instituciones de salud de nuestra región Lambayeque; por lo que, la responsabilidad frente a esta pandemia se traslada a la población quienes deben cumplir con todas las medidas de protección que se promocionan por los canales de comunicación (4).

En nuestro país gran parte de la población ha sido afectada por Covid-19, con manifestaciones clínicas variadas, desde casos asintomáticos hasta aquellos con síntomas leves, moderados y severos. Las secuelas Post COVID - 19 se dan al salir de alta post infección, presentan secuelas que van desde manifestaciones respiratorias, cardíacas, neurológicas y otras que 
requieren de un seguimiento médico multidisciplinario a fin de lograr el retorno a sus actividades (5).

Los pacientes que tuvieron estancias hospitalarias prolongadas requerían seguimiento médico permanente, así como una dieta individualizada para reponer los déficits nutricionales, por la pérdida de peso y masa muscular. Además, hay que prestar especial cuidado a las secuelas con compromiso de piel (úlceras de presión en rostro, tronco y extremidades) que pudieran presentar estos pacientes por la hospitalización prolongada, ya que pueden requerir un manejo médico estricto y continuos cuidados para evitar complicaciones mayores. Es importante que la evaluación médica post infección pueda identificar también síntomas de depresión, ansiedad o estrés postraumático, que pueden requerir tratamiento farmacológico, terapia de apoyo psicológico y soporte familiar para recuperar la estabilidad mental (5).

Es por ello que formularon como problema general: ¿Cómo se relaciona los Factores de Comorbilidad y Secuelas de Covid 19 en trabajadores de un Hospital Nacional de Huancayo 2021? Y como problemas específicos, ¿Cuáles son los factores de Comorbilidad de Covid 19 en trabajadores de un Hospital Nacional de Huancayo 2021?, ¿Cuáles son las Secuelas de Covid 19 en trabajadores de un Hospital Nacional de Huancayo 2021?

El objetivo general es determinar la relación de los Factores de Comorbilidad y Secuelas de Covid 19 en trabajadores de un Hospital Nacional de Huancayo 2021; asi mismo los objetivos específicos: identificar los factores de Comorbilidad de Covid 19 en trabajadores de un Hospital Nacional de Huancayo 2021, evaluar las Secuelas de Covid 19 en trabajadores de un Hospital Nacional de Huancayo 2021.

La conveniencia de este estudio es debido a los distintos cambios organizativos a nivel político, social, económico y tecnológico causado por la situación mundial emergente del COVID-19, tomando en cuenta que esta situación de emergencia no se ha vivido antes y ha causado impacto en todos los niveles organizativos del país. Debido a esto se ha evidenciado diferentes estudios donde se toma en cuenta tanto la comorbilidad como las secuelas y repercusiones que tienen el estrés, la depresión y ansiedad en el trabajo, en la salud y en la economía global. Se justifica teóricamente debido a las estimaciones realizadas por parte de la Organización Mundial de la Salud en el mundo hay 264 millones de personas con secuelas como la depresión, una de las principales Con este estudio se busca proponer un plan con medidas preventivas que se puedan efectuar en situaciones similares a la ocasionada por el COVID-19, ofreciendo a los trabajadores condiciones óptimas en su lugar de trabajo, tomando en cuenta la satisfacción y el bienestar emocional lo que se traducirá en resultados positivos a la organización y su progreso se verá reflejado con el cumplimiento de los objetivos planteados. En la práctica esta investigación identificará la comorbilidad y las secuelas a causa del COVID - 19 en el personal de salud en esta parte del país, resultados que podrán ser utilizados para tomar medidas a nivel de los establecimientos de salud en beneficio de los trabajadores. En el aspecto social este estudio proporcionará adecuada información sobre el riesgo de mortalidad al inicio de la atención de soporte de los pacientes que son trabajadores del sector salud, teniendo en consideración factores clínicos y demográficos que pueden ser aplicables a cualquier realidad. Se recopilará información desde la admisión hasta el alta como fallecido o sobreviviente de la enfermedad. Por lo tanto, estos datos proporcionan un marco importante para construir estrategias de atención de pacientes en la lucha contra esta pandemia de todas las personas de la sierra central y por extensión del Perú. La justificación metodológica es porque para esta investigación se elaborará un instrumento de recolección de datos dirigido al personal de salud con indicadores de comorbilidad y de secuelas del COD - 19, instrumento que será validado por un juicio de expertos y servirán de aporte para investigaciones posteriores en un contexto similar.

Se tiene como antecedente para la Variable Factores de comorbilidad a Díaz F. y Toro, A. en la investigación SARS-CoV-2/COVID-19 el virus, la enfermedad y la pandemia, 2020; para el artículo Editora Médica Colombiana S.A.; cuyo objetivo es revisar el virus causante de esta nueva pandemia COVID-19 que afecta al mundo; acerca de la metodología es descriptiva y documental; los resultados evidenciaron que los datos recogidos hasta el momento indican que la COVID-19 es una enfermedad causada por un coronavirus altamente transmisible, con una tasa de letalidad entre baja y moderada, dependiendo de las comorbilidades y la situación geográfica. Concluyendo que las esperanzas a corto plazo están puestas en el desarrollo de una prueba serológica óptima, con la suficiente especificidad diagnóstica para que diferencie el SARSCoV-2 de los otros coronavirus (6).

Y como antecedente para la Variable Secuelas del COVID 19 se tiene a Andrade M. Campo D. Díaz V. 
Flórez D. Mayorca D. Ortiz A. Ramírez S. Ruiz C. Santos S. Santofinio A. y Yosa A. en la tesis Descripción de las características psicológicas relacionadas con la salud mental en la situación de emergencia de salud pública originada por el COVID-19, 2020; para optar el título de Psicólogos en la Universidad Cooperativa de Colombia; Neiva Colombia; cuyo objetivo es describir las características relacionadas con la salud mental en la comunidad vinculada a una institución educativa universitaria de carácter privado, durante el aislamiento preventivo obligatorio por el COVID-19; acerca de la metodología es descriptivo, cuantitativo; los resultados evidenciaron que se contó con la participación de 174 personas que presentan algún vínculo con la Universidad Cooperativa de Colombia; referente a las características sociodemográficas y laborales de los participantes del estudio, se contó con una participación predominante de mujeres, especialmente jóvenes que se encuentran realizando estudios universitarios, razón por la cual, es probable que sean personas que dependen económicamente de terceros como padres u otros familiares En cuanto a la identificación de las condiciones familiares y conductuales de los participantes, durante la medida de aislamiento preventivo obligatorio; asimismo otros de los resultados del estudio exponen síntomas como dolores de cabeza, pérdida del apetito, insomnio, malestar estomacal; temores de infección, aburrimiento y pensamiento suicida. Concluyendo que el estar confinados, con mínimos momentos semanales para movilizarse con libertad, pero controlados con una serie de estrategias, ha marcado la estabilidad física y mental de la población investigada (7).

En la base teórica desarrollamos lo siguiente: Comorbilidad para COVID 19 es el Sobrepeso obesidad. La obesidad y el sobrepeso se definen como una acumulación anormal o excesiva de grasa que puede ser perjudicial para la salud. Una forma simple de medir la obesidad es el índice de masa corporal (IMC), esto es el peso de una persona en kilogramos dividido por el cuadrado de la talla en metros. Una persona con un IMC igual o superior a 30 es considerada obesa y con un IMC igual o superior a 25 es considerada con sobrepeso. El sobrepeso y la obesidad son factores de riesgo para numerosas enfermedades crónicas, entre las que se incluyen la diabetes, las enfermedades cardiovasculares y el cáncer (3). También el cáncer que es un proceso de crecimiento y diseminación incontrolados de células. Puede aparecer prácticamente en cualquier lugar del cuerpo. El tumor suele invadir el tejido circundante y puede provocar metástasis en puntos distantes del organismo. Muchos tipos de cáncer se podrían prevenir evitando la exposición a factores de riesgo comunes como el humo de tabaco. Además, un porcentaje importante de cánceres pueden curarse mediante cirugía, radioterapia o quimioterapia, especialmente si se detectan en una fase temprana (3). La Diabetes que es una enfermedad crónica que aparece cuando el páncreas no produce insulina suficiente o cuando el organismo no utiliza eficazmente la insulina que produce. El efecto de la diabetes no controlada es la hiperglucemia (aumento del azúcar en la sangre) (3). Otro factor de comorbilidad es el VIH, el virus de la inmunodeficiencia humana (VIH) infecta a las células del sistema inmunitario, alterando o anulando su función. La infección produce un deterioro progresivo del sistema inmunitario, con la consiguiente "inmunodeficiencia". Se considera que el sistema inmunitario es deficiente cuando deja de poder cumplir su función de lucha contra las infecciones y enfermedades. El síndrome de inmunodeficiencia adquirida (SIDA) es un término que se aplica a los estadios más avanzados de la infección por VIH y se define por la presencia de alguna de las más de 20 infecciones oportunistas o de cánceres relacionados con el VIH (3). También la cardiopatía, las enfermedades cardiovasculares (ECV) son un conjunto de trastornos del corazón y de los vasos sanguíneos. Son la principal causa de defunción en todo el mundo (3). El asma, que es una de las principales enfermedades no transmisibles. Se trata de una enfermedad crónica que provoca inflamación y estrechamiento de las vías que conducen el aire a los pulmones (3), neumopatía crónica, la enfermedad pulmonar obstructiva crónica (EPOC) se caracteriza por un bloqueo persistente del flujo de aire. Se trata de una enfermedad subdiagnosticada y potencialmente mortal que altera la respiración normal y no es totalmente reversible (3).

Las secuelas Covid 19, como parte del proceso fisiopatológico de la COVID-19, se genera una repuesta inflamatoria intensa que tiene al tracto respiratorio y principalmente el pulmón como primer órgano afectado. Sin embargo, varios estudios apuntan que las secuelas de esta infección no sólo se limitan al aparato respiratorio, y que se han registrado secuelas en el sistema cardiovascular, y en el sistema nervioso central y periférico. Se ha documentado también secuelas psiquiátricas y psicológicas (8). Secuelas en el aparato respiratorio, los pacientes que desarrollaron un cuadro clínico grave de COVID-19 tienen como principal secuela el desarrollo de fibrosis pulmonar. Durante la fase aguda de la infección por SARS- CoV-2, el daño pulmonar causa edema, desprendimiento alveolar de 
células epiteliales y depósito de material hialino en las membranas alveolares. Durante la siguiente fase de la infección, que usualmente ocurre entre las segunda y quinta semana, los pulmones muestran signos de fibrosis, con el depósito de fibrina y la infiltración de células inflamatorias y fibroblastos cerca de las células epiteliales, en los espacios alveolares. Durante la etapa final, entre la sexta y octava semanas, el tejido pulmonar se vuelve fibrótico. Adicionalmente, hay varios reportes de lesiones bilaterales con predominio del lóbulo inferior (8). Secuelas en el sistema cardiovascular, se ha documentado que pacientes con formas graves de COVID-19 presentaron lesiones significativas de miocardio, incluyendo miocarditis relacionada a infección, con reducción de la función sistólica y arritmias. Estas lesiones podrían ser secundarias al daño pulmonar severo.

\section{MATERIAL Y MÉTODOS}

De acuerdo a Tamayo "el método de esta investigación científico consiste en un método de investigación usado principalmente en la producción de conocimiento en las ciencias. Para ser llamado científico, un método de investigación debe basarse en la empírica y en la medición" (9)

De acuerdo a la finalidad realizada es básica, porque mejora el conocimiento y comprensión de los fenómenos sociales, así mismo es el fundamento de toda investigación. (10)

De acuerdo a Hernández R, Fernández C, y Baptista $\mathrm{P}$, "el nivel de investigación es correlacional, es decir que luego de conocer la situación problemática y determinar su frecuencia, podemos determinar qué factores están relacionados con ésta frecuencia registrada" (11).

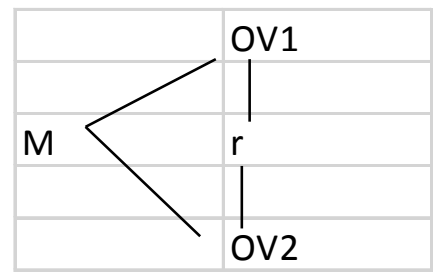

El diseño es correlacional, no experimental y de corte transversal. El esquema es:

\section{Dónde:}

$\mathrm{M}=$ Muestra:

OV1 = Observación de la variable 1 :

OV2 = Observación de la Variable 2:

$\mathrm{r}=$ Correlación entre la variable 1 y la variable 2

\section{Población}

De acuerdo a Hernández R, Fernández C, y Baptista P, "se llama así a la totalidad de individuos o elementos quienes presenta una característica en común susceptible de estudio, pero que no siempre es posible estudiarlo en su totalidad". (11), En este caso la población está conformada por 216 trabajadores de salud en el hospital de estudio que tuvieron COVID 19 entre el 2020 y 2021

\section{Criterios de Inclusión}

Los trabajadores del Hospital en estudio que tuvieron COVID - 19 .

\section{Criterios de Exclusión}

Trabajadores del hospital que se encuentren en licencia, vacaciones.

\section{Muestra}

Según (Sánchez H, y Reyes C, "una muestra es un subconjunto de la población, que se obtiene para averiguar las propiedades o características de esta última, por lo que interesa que sea un reflejo de la población, que sea representativa de ella, concepto al que volveremos más adelante" (12). La muestra estuvo conformada por 137 trabajadores de salud en el hospital de estudio que tuvieron COVID - 19.

El tamaño de muestra se obtuvo utilizando la siguiente formula.

$$
n=\frac{Z^{2} N \times p \times q}{(N-1) \times E^{2}+Z^{2} \cdot p \times q}
$$

$\mathrm{n}=$ tamaño de la muestra que se desea saber

$\mathrm{Z}=$ nivel de confianza $(95 \%=1.96)$

$\mathrm{N}=$ representa el tamaño de la población $=216$

$\mathrm{p}=$ probabilidad a favor $(0.5)$

$\mathrm{q}=$ probabilidad en contra $(0.5)$

$\mathrm{E}=$ error de estimación $(0.05)$

$$
\begin{gathered}
n=\frac{1.96^{2} .216 \times 0.5 \times 0.5}{(216-1) \times 0.05^{2}+1.96^{2} .0 .5 \times 0.5} \\
n=137
\end{gathered}
$$

\section{Técnicas e instrumentos de recolección de datos}

De acuerdo a Kerlinger "la técnica de recopilación de datos de la encuesta que utiliza un conjunto de 
procedimientos estandarizados de investigación mediante los cuales se recoge y analiza una serie de datos de una muestra de casos representativa de una población o universo más amplio, del que se pretende explorar" (10). Esta investigación tuvo como técnica la encuesta y como instrumento el cuestionario

\section{Validez}

Para, Sánchez y Reyes "la validez es la propiedad que hace referencia a que todo instrumento debe medir lo que se ha propuesto medir, vale decir que demuestre efectividad al obtener los resultados de la capacidad, conducta, rendimiento o aspectos que asegura medir. En este caso se validará el instrumento por el juicio de expertos" (12).

\section{Confiabilidad}

La confiabilidad del instrumento fue probada con el coeficiente alfa de cronbach por tener ambos instrumentos alternativos de respuesta múltiple. De acuerdo a Rosas y Zúñiga se considera el instrumento confiable a partir de un valor de 0,75 (13). En este estudio el cuestionario de factores de comorbilidad para una prueba piloto de 30 y 14 ítems tiene un valor de alfa de Cronbach de 0,952 y el cuestionario de secuelas de COVID-19 para una prueba piloto de 30 y cinco ítems tiene un valor de 0,770 . Por lo tanto, ambos instrumentos son confiables.

\section{RESULTADOS}

La tabla 1 muestra los datos generales de la muestra que estuvo compuesta por 137 trabajadores de salud en el hospital de estudio que tuvieron COVID - 19 entre el 2020 y 2021. Fueron la mayoría profesionales en $52,6 \%$. Tuvieron COVID-19 la mayoría leve $(51,8 \%)$, sí tuvieron contacto con los pacientes el $81,8 \%$ y cree que se contagió en el hospital en 58,4\% y recibió atención en su casa el 43,8\% y en un hospital general el $29,9 \%$.

Se puede ver en la tabla 2 que la mayoría tenía entre 31 y 40 años $(35,8 \%)$ seguido de 41 y 50 años $(30,7)$, fueron de sexo masculino $(60,6 \%)$. Entre los factores de comorbilidad tenían: sobrepeso el $65 \%$, cáncer el $0 \%$, diabetes el $8 \%$, VIH $0 \%$, cardiopatías el 3\% asma al 10,2\%, enfermedades pulmonares obstructivo crónico el $0 \%$, enfermedades del hígado $19,7 \%$ trastorno hematológico el 3,6\%, hipertensión

Tabla 1. Datos generales.

\begin{tabular}{lcc}
\hline \multicolumn{1}{c}{ ÍTEMS } & $\begin{array}{c}\text { FRECUENCIA } \\
(\mathbf{n}=\mathbf{1 3 7})\end{array}$ & PORCENTAJE \\
\hline 1. Grupo profesional a que pertenece & 72 & $52,6 \%$ \\
Profesional & 65 & $47,4 \%$ \\
No profesional & & \\
2. ¿En qué grado se presentó el COVID 19? & 71 & $51,8 \%$ \\
Leve & 46 & $33,6 \%$ \\
Moderado & 20 & $14,6 \%$ \\
Severo & 25 & \\
3. Ud. Estuvo en contacto con pacientes COVID 19, por motivos de su trabajo: & $18,2 \%$ \\
No & 112 & $81,8 \%$ \\
Si & $25 \%$ \\
4. Ud. Cree que se contagió durante el desempeño de sus actividades dentro del hospital & \\
No & 57 & $41,6 \%$ \\
Si & 80 & $58,4 \%$ \\
5. Recibió atención de salud, al ser diagnosticado de COVID 19 en: & \\
Hospital Especializado & 4 & $2,9 \%$ \\
Hospital General & 41 & $29,9 \%$ \\
Clínica & 10 & $7,3 \%$ \\
Centro de Salud & 19 & $13,9 \%$ \\
Puesto de salud & 3 & $2,2 \%$ \\
Cuidaos En Casa & 60 & $43,8 \%$ \\
\hline
\end{tabular}


Tabla2.Factores de comorbilidad

\begin{tabular}{|c|c|c|}
\hline ÍTEMS & $\begin{array}{l}\text { FRECUENCIA } \\
(n=137)\end{array}$ & PORCENTAJE \\
\hline \multicolumn{3}{|l|}{ 1. $\quad$ Edad } \\
\hline De 20 a 30 años & 21 & $15,3 \%$ \\
\hline De 31 a 40 años & 49 & $35,8 \%$ \\
\hline De 41 a 50 años & 42 & $30,7 \%$ \\
\hline De 51 a 60 años & 21 & $15,3 \%$ \\
\hline \multicolumn{3}{|l|}{ 2. Sexo } \\
\hline Femenino & 54 & $39,4 \%$ \\
\hline Masculino & 83 & $60,6 \%$ \\
\hline \multicolumn{3}{|l|}{ 3. Sobrepeso } \\
\hline No & 48 & $35,0 \%$ \\
\hline $\mathrm{Si}$ & 89 & $65,0 \%$ \\
\hline \multicolumn{3}{|l|}{ 4. Cáncer } \\
\hline No & 137 & $100 \%$ \\
\hline $\mathrm{Si}$ & 0 & $0 \%$ \\
\hline \multicolumn{3}{|l|}{ 5. Diabetes } \\
\hline No & 126 & $92 \%$ \\
\hline $\mathrm{Si}$ & 11 & $8.0 \%$ \\
\hline \multicolumn{3}{|l|}{ 6. VIH } \\
\hline No & 137 & $100 \%$ \\
\hline $\mathrm{Si}$ & 0 & $0 \%$ \\
\hline \multicolumn{3}{|l|}{ 7. Cardiopatías } \\
\hline No & 133 & $97 \%$ \\
\hline $\mathrm{Si}$ & 4 & $3 \%$ \\
\hline \multicolumn{3}{|l|}{ 8. Asma } \\
\hline No & 123 & $89.8 \%$ \\
\hline $\mathrm{Si}$ & 14 & $10.2 \%$ \\
\hline \multicolumn{3}{|c|}{ 9. Enfermedades pulmonares obstructivo crónico } \\
\hline No & 137 & $100 \%$ \\
\hline $\mathrm{Si}$ & 0 & $0 \%$ \\
\hline \multicolumn{3}{|c|}{ 10. Enfermedades del hígado } \\
\hline No & 110 & $80.3 \%$ \\
\hline $\mathrm{Si}$ & 27 & $19.7 \%$ \\
\hline \multicolumn{3}{|l|}{ 11. Trastorno Hematológico } \\
\hline No & 132 & $96.4 \%$ \\
\hline $\mathrm{Si}$ & 5 & $3.6 \%$ \\
\hline \multicolumn{3}{|l|}{ 12. Hipertensión Arterial } \\
\hline No & 78 & $56.9 \%$ \\
\hline $\mathrm{Si}$ & 59 & $43.1 \%$ \\
\hline \multicolumn{3}{|c|}{ 13. Enfermedades Neurológicas } \\
\hline No & 137 & $100 \%$ \\
\hline $\mathrm{Si}$ & 0 & $0 \%$ \\
\hline \multicolumn{3}{|l|}{ 14. Enfermedades Renales } \\
\hline No & 135 & $98.5 \%$ \\
\hline $\mathrm{Si}$ & 2 & $1.5 \%$ \\
\hline
\end{tabular}


Tabla 3. Secuelas Post Covid -19

\begin{tabular}{|c|c|c|}
\hline ÍTEMS & $\begin{array}{c}\text { FRECUENCIA } \\
(\mathrm{n}=137)\end{array}$ & PORCENTAJE \\
\hline \multicolumn{3}{|l|}{ 1. Secuelas en el aparato respiratorio } \\
\hline Ninguno & 56 & $40,9 \%$ \\
\hline Fibrosis pulmonar & 2 & $1,5 \%$ \\
\hline Disnea & 0 & $0 \%$ \\
\hline Cansancio o fatiga & 43 & $31,4 \%$ \\
\hline Tos & 28 & $20,4 \%$ \\
\hline Dificultad para respirar o falta de aire & 8 & $5,8 \%$ \\
\hline \multicolumn{3}{|c|}{ 2. Secuelas en el sistema cardiovascular } \\
\hline Ninguno & 119 & $86,9 \%$ \\
\hline Miocarditis & 0 & $0 \%$ \\
\hline Tromboembolismo & 0 & $0 \%$ \\
\hline Arritmias & 4 & $2,9 \%$ \\
\hline Hipertensión arterial & 14 & $10,2 \%$ \\
\hline \multicolumn{3}{|l|}{ 3. Secuelas neuropsiquiatrías } \\
\hline \multicolumn{3}{|l|}{ Pérdida de olfato } \\
\hline No & 131 & $95,6 \%$ \\
\hline $\mathrm{Si}$ & 6 & $4,4 \%$ \\
\hline \multicolumn{3}{|l|}{ Cefalea } \\
\hline No & 68 & $49,6 \%$ \\
\hline $\mathrm{Si}$ & 69 & $50,4 \%$ \\
\hline \multicolumn{3}{|l|}{ Deterioro cognitivo } \\
\hline No & 137 & $100 \%$ \\
\hline $\mathrm{Si}$ & 0 & $0 \%$ \\
\hline \multicolumn{3}{|l|}{ Cambios de humor } \\
\hline No & 116 & $84,7 \%$ \\
\hline $\mathrm{Si}$ & 21 & $15,3 \%$ \\
\hline \multicolumn{3}{|l|}{ Disfunción neuromuscular } \\
\hline No & 136 & $99,3 \%$ \\
\hline $\mathrm{Si}$ & 1 & $0,7 \%$ \\
\hline \multicolumn{3}{|l|}{ Dificultad para pensar o concentrarse } \\
\hline No & 125 & $91,2 \%$ \\
\hline $\mathrm{Si}$ & 12 & $8,8 \%$ \\
\hline \multicolumn{3}{|l|}{ Depresión } \\
\hline No & 81 & $59,1 \%$ \\
\hline $\mathrm{Si}$ & 56 & $40,9 \%$ \\
\hline \multicolumn{3}{|l|}{ Ansiedad } \\
\hline No & 124 & $90,5 \%$ \\
\hline $\mathrm{Si}$ & 13 & $9,5 \%$ \\
\hline \multicolumn{3}{|l|}{ Dolor muscular } \\
\hline No & 123 & $89,8 \%$ \\
\hline $\mathrm{Si}$ & 14 & $10,2 \%$ \\
\hline
\end{tabular}


Tabla 3. (cont.) Secuelas Post Covid -19

\begin{tabular}{lcr}
\hline \multicolumn{1}{c}{ ÍTEMS } & $\begin{array}{c}\text { FRECUENCIA } \\
(\mathbf{n}=\mathbf{1 3 7})\end{array}$ & PORCENTAJE \\
\hline Pérdida de la memoria & 137 & $100 \%$ \\
$\mathrm{No}$ & 0 & $0 \%$ \\
$\mathrm{Si}$ & & \\
4. Secuelas psicológicas & 122 & $89,1 \%$ \\
Ninguno & 15 & $10,9 \%$ \\
Negativismo & & \\
5. Dermatológico & 65 & $47,4 \%$ \\
Ninguno & 59 & $43,1 \%$ \\
Caída de cabello & 13 & $9,5 \%$ \\
Urticarias & & \\
&
\end{tabular}

Tabla 4. Rho de Spearman para factores de comorbilidad y secuela post Covid 19

\begin{tabular}{clcc}
\hline \multicolumn{1}{c}{ Correlaciones } & & \\
\hline & & $\begin{array}{c}\text { Factores de } \\
\text { Comorbilidad }\end{array}$ & Secuelas \\
\hline Factores de & Correlación de Pearson & 1 & $0,229^{* *}$ \\
Comorbilidad & Sig. (bilateral) & & 0,007 \\
& $\mathrm{~N}$ & 137 & 137 \\
& Correlación de Pearson & $0,229^{* *}$ & 1 \\
Secuelas & Sig. (bilateral) & 0,007 & 137 \\
& $\mathrm{~N}$ & 137 & 137 \\
\hline
\end{tabular}

**. La correlación es significativa en el nivel 0,01 (bilateral).

arterial el 43,1\%., enfermedades neurológicas el $0 \%$ y enfermedades renales el 1,5\%.

En cuanto a las secuelas la tabla 3 muestra que la mayoría no tenía secuelas del aparato respiratorio $(40,9 \%)$, tampoco cardiovasculares $(86,9 \%)$, ni secuelas psicológicas $(89,1 \%)$ tampoco dermatológicas $(47,4 \%)$. En relación a las secuelas neuropsiquiátricas se observa que tuvieron pérdida de olfato el $4,4 \%$, cefalea el 50,4\%; deterioro cognitivo, ninguno; cambios de humor $15,3 \%$; disfunción neuromuscular $0,7 \%$; dificultad para pensar o concentrarse el $8,8 \%$; depresión 40,9\%; ansiedad 9,5\%; dolor muscular $10,2 \%$; pérdida de la memoria: ninguno.

\section{Prueba de Hipótesis}

$\mathrm{H} 0=$ No existe relación entre los factores de Comorbilidad y secuelas relacionados con el Covid 19 en trabajadores de un Hospital Nacional de Huancayo 2021.

$\mathrm{HI}=$ Existe relación entre los factores de Comorbilidad y secuelas relacionados con el Covid 19 en trabajadores de un Hospital Nacional de Huancayo 2021.

Nivel de significancia: $\alpha=0,05$

El valor de la correlación Pearson es 0,229 (tabla 4), lo que nos indica que la correlación es directa y baja y la significancia bilateral $(\mathrm{p}$ valor $=0,007<0,05$ )

Por lo tanto, se rechaza la hipótesis nula y se acepta la de investigación en el sentido que existe relación entre los factores de Comorbilidad y secuelas relacionados con el Covid 19 en trabajadores de un Hospital Nacional de Huancayo 2021 ( $p$ valor $=0.007<0.05$ ).

\section{DISCUSIÓN}

Los resultados del objetivo general muestran existe relación entre los factores de Comorbilidad y secuelas relacionados con el Covid 19 en trabajadores de un Hospital Nacional de Huancayo 2021 (p valor $=0,007<0,05$ ). Donde la muestra que estuvo compuesta por 137 trabajadores de salud en el hospital de estudio que tuvieron COVID - 19 entre el 2020 
y 2021. Fueron la mayoría profesionales en $52,6 \%$. Tuvieron COVID-19 la mayoría leve $(51,8 \%)$ y severo el $14,6 \%$, sí tuvieron contacto con los pacientes el $81,8 \%$ y cree que se contagió en el hospital en $58,4 \%$ y recibió atención en su casa el $43,8 \%$ y en un hospital general el $29,9 \%$. Los resultados del objetivo específico 1 muestran que los factores de comorbilidad donde la mayoría tenía entre 31 y 40 años $(35,8 \%)$ seguido de 41 y 50 años $(30,7)$, fueron de sexo masculino $(60,6 \%)$. Entre los factores de comorbilidad tenían: sobrepeso el $65 \%$, cáncer el $0 \%$, diabetes el $8 \%$, VIH $0 \%$, cardiopatías el $3 \%$ asma al $10,2 \%$, enfermedades pulmonares obstructivo crónico el $0 \%$, enfermedades del hígado 19,7\% trastorno hematológico el 3,6\%, hipertensión arterial el 43,1\%., enfermedades neurológicas el $0 \%$ y enfermedades renales el 1,5\%. Los resultados del objetivo específico 2 muestran que las secuelas la tabla muestra que la mayoría no tenía secuelas del aparato respiratorio (40,9\%), tampoco cardiovasculares $(86,9 \%)$, ni secuelas psicológicas $(89,1 \%)$ tampoco dermatológicas $(47,4 \%)$. En relación a las secuelas neuropsiquiátricas se observa que tuvieron pérdida de olfato el $4,4 \%$, cefalea el 50,4\%; deterioro cognitivo, ninguno; cambios de humor $15,3 \%$; disfunción neuromuscular $0,7 \%$; dificultad para pensar o concentrarse el 8,8\%; depresión $40,9 \%$; ansiedad 9,5\%; dolor muscular 10,2\%; pérdida de la memoria, ninguno.

Un estudio que difiere de este en sus resultados fue el de Díaz F. y Toro, A. cuyos los resultados evidenciaron que las esperanzas a corto plazo están puestas en el desarrollo de una prueba serológica óptima, con la suficiente especificidad diagnóstica para que diferencie el SARS-CoV-2 de los otros coronavirus (6).

En relación a las secuelas un estudio que difiere de este es el de Andrade M. Campo D. et.al. quienes encontraron que los trabajadores del área de cajas del hospital que han sido diagnosticados con alguna enfermedad preexistente, se encuentran dentro del grupo más vulnerable de contraer COVID-19 y enfermarse gravemente, por ende, genera situaciones abrumadoras y de temor influyendo en su salud mental y física de los mismos.

Por su lado la teoría refiere los factores de comorbilidad predisponen a una neumonía COVID (3), sin embargo en esta investigación la mayoría de los trabajadores tuvo síntomas leves en el $51,8 \%$ y solo síntomas severos el 14,6\%. Y también la teoría señala que las Secuelas Covid 19, son parte del proceso fisiopatológico de la COVID-19, se genera una repuesta inflamatoria en diferentes partes $\mathrm{u}$ órganos del cuerpo. Sin embargo, en esta investigación se pudo comprobar que la mayoría de los trabajadores del Hospital en estudio no tienen secuelas la mayoría de ellos.

\section{CONCLUSIONES}

Se ha determinado que existe relación entre los factores de Comorbilidad y secuelas relacionados con el Covid 19 en trabajadores de un Hospital Nacional de Huancayo 2021 ( $p$ valor $=0,007<0,05$ ). Donde la muestra que estuvo compuesta por 137 trabajadores de salud en el hospital de estudio que tuvieron COVID - 19 entre el 2020 y 2021. Fueron la mayoría profesionales en 52.6\%. Tuvieron COVID-19 la mayoría leve $(51,8 \%)$ y severo el $14,6 \%$, sí tuvieron contacto con los pacientes el $81.8 \%$ y cree que se contagió en el hospital en 58,4\% y recibió atención en su casa el $43.8 \%$ y en un hospital general el $29.9 \%$.

Se ha identificado los factores de comorbilidad donde la mayoría tenía entre 31 y 40 años $(35,8 \%)$ seguido de 41 y 50 años (30,7\%), fueron de sexo masculino $(60,6 \%)$. Entre los factores de comorbilidad tenían: sobrepeso el $65 \%$, cáncer el $0 \%$, diabetes el $8 \%$, VIH $0 \%$, cardiopatías el $3 \%$ asma al $10,2 \%$, enfermedades pulmonares obstructivo crónico el $0 \%$, enfermedades del hígado 19,7\% trastorno hematológico el 3,6\%, hipertensión arterial el 43.1\%., enfermedades neurológicas el $0 \%$ y enfermedades renales el $1,5 \%$.

Se ha identificado las secuelas la tabla muestra que la mayoría no tenía secuelas del aparato respiratorio (40,9\%), tampoco cardiovasculares $(86,9 \%)$, ni secuelas psicológicas $(89 ., 1 \%)$ tampoco dermatológicas $(47,4 \%)$. En relación a las secuelas neuropsiquiátricas se observa que tuvieron pérdida de olfato el $4,4 \%$, cefalea el $50,4 \%$; deterioro cognitivo, ninguno; cambios de humor 15,3\%; disfunción neuromuscular $0,7 \%$; dificultad para pensar o concentrarse el 8,8\%; depresión 40,9\%; ansiedad $9,5 \%$; dolor muscular $10,2 \%$; pérdida de la memoria, ninguno.

\section{Correspondencia:}

Anahi Indira De La Calle Castro

Correo electrónico: adelacalle@uroosevelt.edu.pe

\section{REFERENCIAS BIBLIOGRAFICAS}

1. Pérez, M. y Gómez, J. Características clínico epidemiológicas de la COVID 19. Rev haban cienc méd. 2020; 19(2):e_3254. (Citado el 15 de marzo 
del 2021) Disponible en: http://scielo.sld.cu/pdf/ rhcm/v19n2/1729-519X-rhcm-19-02-e3254.pdf

2. Herrera D. Características clínicas y epidemiológicas de la COVID-19 en pediatría en República Dominicana.Revista Cubana de Pediatria. 2021; 93(1). (Citado el 15 de marzo del 2021) Disponible en: http://www.revpediatria.sld.cu/index.php/ped/ article/view/1319

3. Organización Mundial de la Salud. Evaluación de los factores de riesgo de enfermedad por el coronavirus de 2019 (COVID-19) entre trabajadores de salud: protocolo para un estudio de casos y testigos. Ginebra: Organización Mundial de la Salud; 2019. (Citado el 15 de marzo del 2021) Disponible en: https://apps. who.int/iris/handle/10665/332505, 2020.

4. Saavedra F. La comorbilidad y el COVID 19. Chiclayo: Universidad Católica Santo Toribio de Mogrovejo; 2020. (Citado el 15 de marzo del 2021) Disponible en:http://www.usat.edu.pe/articulos/la-comorbilidad$\mathrm{y}-\mathrm{e} 1$ - covid - 19/\#: : t ext $=\mathrm{Cu}$ and o $\% 20$ mencionamos $\% 20$ morbilidad $\% 20$ en $\% 20$ $\mathrm{t} \% \mathrm{C} 3 \% \mathrm{~A} 9 \mathrm{rminos}$,mismo\%20tiempo\%20o\%20 uno $\% 20$ despu $\% \mathrm{C} 3 \% \mathrm{~A} 9 \mathrm{~s}$

5. Alva D. El manejo de las secuelas Post Covid 19. Lima, Perú: Clinica San Felipe; 2020. (Citado el 15 de marzo del 2021) Disponible en: https://www. clinicasanfelipe.com/articulos/manejo-las-secuelaspost-covid-19

6. Díaz F, Toro A. SARS-CoV-2/COVID-19 el virus, la enfermedad y la pandemia. Bogota: Editora Médica Colombiana SA; 2020.

7. Andrade M. Descripción de las características psicológicas relacionadas con la salud mental en la situación de emergencia de salud pública originada por el COVID-19. Tesis de Grado para optar el título de Psicólogos. Neiva Colombia: Universidad Cooperativa de Colombia; 2020.

8. Organización Panamericana de la Salud. Alerta epidemiológica - complicaciones y secuelas por COVID -19. Washington DC: Organización Panamericana de la Salud; 2020.

9. Tamayo M. El proceso de la investigación científica. Ciudad de México: Limusa Noriega editores; 2000.
10. Kerlinger FN. Enfoque conceptual de la investigación del comportamiento. Ciudad de México: Editorial Interamerican; 2002.

11. Hernández R, Fernández C, Baptista P. Metodología de la investigación. Bogota: Editorial Mc. Graw Hill; 2014.

12. Sánchez H, Reyes C, Sánchez H, Reyes C. Metodología y diseños en la investigación científica. Lima: Universitaria; 2002.

13. Rosas y Zúñiga A. Estadística Descriptiva e Inferencial I. Correlación y regresión lineales. Ciudad de Mexico: Colegio de Bachilleres; 2010.

14. Ortiz K. El impacto de los factores de riesgo en la motivación laboral del personal de salud en la crisis por covid-19. Tesis para optar el título de licenciada en Ciencias Administrativas. Piura, Perú: Universidad Nacional de Piura; 2020.

15. Culquichicón C. Factores de riesgo asociados a infección severa y muerte por neumonía de coronavirus-19 en pacientes del seguro social de salud. IETSI; 2020.

16. Mejía F .Características clínicas y factores asociados a mortalidad en pacientes adultos hospitalizados por COVID-19 en un hospital público de Lima, Perú. Heredia, Lima, Perú: Universidad Peruana Cayetano; 2020.

17. Organización de las Naciones Unidas. COVID-19 y la necesidad de actuar en relación con la salud mental. Informe de Políticas de las Naciones Unidas. Washington DC: Organización de las Naciones Unidas; 2020.

18. MedlinePlus. Problemas de la sangre. MedlinePlus; 2019. (Citado el 15 de marzo del 2021) Disponible en: https://medlineplus.gov/spanish/blooddisorders. $\mathrm{html}$

19. Organización Nacional de Transplantes. Promoción de la donación. Madrid: Organización Nacional de Transplantes (Citado el 15 de marzo del 2021) Disponible en: en:http://www.ont.es/informacion/ Paginas/PromociondelaDonacion.aspx

Recibido: $12 / 08 / 2021$

Aceptado: 18/11/2021 